\title{
The more-or-less morphing face illusion: A case of fixation-dependent modulation
}

\author{
Rob van Lier, Arno Koning \\ Radboud University Nijmegen, Donders Institute for Brain, Cognition and Behaviour, \\ Centre for Cognition, Montessorilaan 3, 6525 HR Nijmegen, The Netherlands; \\ e-mail: r.vanlier@donders.ru.nl \\ Received 31 January 2014, in revised form 11 September 2014
}

\begin{abstract}
A visual illusion is presented in which the perceived changes in a morphing sequence depend on eye movements. The phenomenon is illustrated using face morphs: when tracking a moving dot superimposed on a face morphing sequence, the changes in the morphing sequence seem rather small, but when the dot stops moving, the perceived extent of morphing suddenly becomes much larger. We explore this phenomenon further and discuss the observed effects.
\end{abstract}

Keywords: eye movements, face perception

It is well known that eye movements affect our perceptions. For example, during saccades we may be blind to considerable changes in the visual display (known as saccadic suppression; for an overview see Henderson \& Hollingworth, 1998), and during smooth pursuit the human visual system seems less sensitive to transient signals (eg Schütz, Delipetkos, Braun, Kerzel, \& Gegenfurtner, 2007). Recently, Suchow and Alvarez (2011) showed that motion on the retina caused a diminished awareness of featural changes of the moving pattern (referred to as 'silencing'). Here, we demonstrate the effect of eye movements on the extent of perceived changes in face morphing sequences, by contrasting fixation on a stationary dot with following a moving dot using smooth pursuit movements. An example is given in movie 1 (http:// dx.doi.org/10.1068/p7704). The reader is invited to follow the instructions in the movie where a morphing between two faces is presented. Superimposed on the face morphing sequence, there is a red dot that has to be fixated. This red dot may move in a circle between the eyes and nose, in which case the dot has to be tracked, or it can be stationary. It should be observed that the degree of change in the morphing sequence appears to differ substantially between the two conditions. When the moving dot is tracked, the extent of morphing appears to be rather small. However, when the dot stops moving but is continued to be fixated, the degree of morphing appears to increase dramatically. Movie 2 shows a second example with different faces.

To investigate these effects further in an exploratory experiment, we created 7 basic faces (FaceGen modeller ${ }^{\circledR}$ ), of which facial properties differed with approximately equal steps (eg the position of the eyebrows was altered systematically) (see figure 1a). Next we created morphing sequences between three pairs of these 7 basic faces (in figure 1a, faces 2 and 4, faces 3 and 5, and faces 4 and 6). Each sequence consisted of 50 frames (going from one face to the other and back). For each face pair the first/last frame in the sequence was balanced. Each presentation started with the observer fixating a red dot, which could be either stationary or moving (in a figure-of-eight trajectory). Next, while the red dot remained visible, a morphing sequence was shown (see figure 1b). A morphing sequence took approximately $5 \mathrm{~s}$ to complete a full cycle, and lasted for 8 cycles. Eight naive observers judged 12 morphing sequences presented in a random fashion (gif animations subtending a visual angle of $\sim 7 \mathrm{deg}$ ). Following each presentation, observers had to indicate the range of faces that appeared to make up the morphing sequence. They could choose from the 7 basic 
faces that appeared on the screen (as in figure 1a). For example, after seeing a morphing sequence between faces 2 and 4, they could respond that the morphing sequence appeared to range from, let's say, face 2 to face 5. In that case the selected morphing range would be $\Delta_{\text {faces }}=3$. Note that the observers were told that all possible extents given these 7 faces could occur during the experiment. A repeated-measures ANOVA with fixation (2) and face pair (3) as independent variables and 'selected morphing range' $\left(\Delta_{\text {faces }}\right)$ as the dependent variable showed only an effect of fixation $\left(F_{1,7}=23.729, p=0.002, \eta_{\mathrm{p}}^{2}=0.77\right)$, with a larger morphing range chosen when fixating a stationary dot as compared with fixating a moving dot (on average $\Delta_{\text {faces }}=2.85$ versus $\Delta_{\text {faces }}=1.19$, respectively; see figure 1c). Testing the selected morphing range against the actual morphing range $\left(\Delta_{\text {faces }}=2\right)$ revealed significant differences for both fixation and tracking conditions $\left(t_{7}=4.837, p=0.002, d=1.710\right.$, and $t_{7}=-3.052, p=0.019, d=1.079$, respectively).

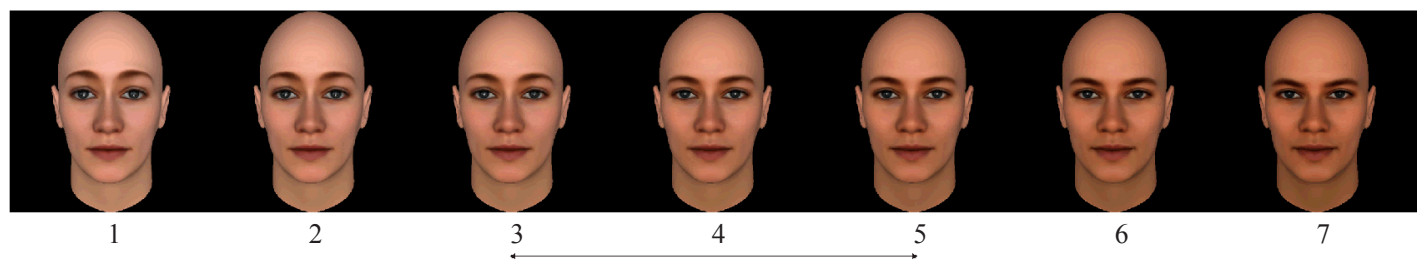

(a)

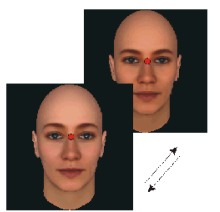

(b)

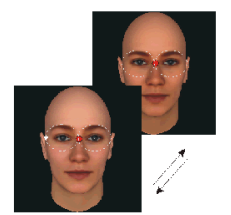

Figure 1. [In colour online, see http://dx.doi.org/10.1068/p7704] (a) The 7 basic faces (see text). The arrows indicate the actual range of faces in the morphing sequences. After each morphing sequence, observers were presented with these 7 faces and had to indicate the range of faces that appeared to make up the morphing sequence. (b) Observers fixated the red dot, which was either stationary (between the eyes) or moved along a figure-of-eight trajectory (indicated here by the white dashed line). (c) Selected morphing range as a function of fixation (stationary versus moving dot), and standard error of the mean. The dashed line indicates the actual morphing range.

What might be the mechanism(s) behind these differential effects? We suggest that the effects in part reflect a modulation of transient signals. For example, the decrease in the apparent morphing range while tracking the moving dot is likely to be due to a reduced sensitivity to transient signals during pursuit eye movements (eg Schütz et al., 2007). We further speculate that the observed increase of the morphing range when fixating the stationary dot might additionally be related to the figural face aftereffect (Carbon \& Ditye, 2011; Tangen, Murphy, \& Thompson, 2011; Webster \& MacLin, 1999). To illustrate the effect of prolonged fixation, the reader may use movie 3 to compare the different percepts interactively. With prolonged and careful fixation of the stationary dot, one might notice that the difference between various properties of the two faces (pale and wrinkly skin together with dark and thick eyebrows versus dark and smooth skin together with light and thin eyebrows) seems to increase with viewing time. In addition, the age gap between the faces may appear to increase (for instructions see the movie caption). 
In sum, we speculate that at least two different mechanisms might be responsible for the reported observations. One involves the attenuated sensitivity to transient signals during pursuit eye movements, and the other involves the amplification of perceptual differences during steady fixation at a single location. Future research, however, is needed to confirm and disentangle these separate effects, by having more variability in the morphs and a more fine-grained response measure, and it may also focus on the face-specificity versus generality of the observed effects. It seems reasonable to expect that similar effects might be obtained for morphs between images other than faces. The relative strength of these effects may then be dependent on the level of familiarity (eg variations in colour may have larger effects for more familiar objects) or expertise. So far, our morphing face illusion shows that perceived face differences can change depending on the way of looking - more or less.

Acknowledgment. This illusion was previously nominated as 'best visual illusion of the year' (2011) by the Neural Correlate Society, and presented at the 'Best Visual Illusion Contest' during the Vision Sciences Society meeting in Naples, FL.

\section{References}

Carbon, C. C., \& Ditye, T. (2011). Sustained effects of adaptation on the perception of familiar faces. Journal of Experimental Psychology: Human Perception and Performance, 37, 615-625.

Henderson, J. M., \& Hollingworth, A. (1998). Eye movements during scene viewing: An overview. In G. Underwood (Ed.), Eye guidance in reading and scene perception (pp. 269-293). Oxford: Elsevier.

Schütz, A. C., Delipetkos, E., Braun, D. I., Kerzel, D., \& Gegenfurtner, K. R. (2007). Temporal contrast sensitivity during smooth pursuit eye movements. Journal of Vision, 7(13):3, 1-15.

Suchow, J. W., \& Alvarez, G. A. (2011). Motion silences awareness of visual change. Current Biology, 21, 140-143.

Tangen, J. M., Murphy, S. C., \& Thompson, M. B. (2011). Flashed face distortion effect: Grotesque faces from relative spaces. Perception, 40, 628-630.

Webster, M. A., \& MacLin, O. H. (1999). Figural aftereffects in the perception of faces. Psychonomic Bulletin \& Review, 6, 647-653. 